offiziell als Sonderassistent firmierenden Allen W. Dulles (überwachte alle OSS-Operationen in Europa) in den Jahren des Zweiten Weltkrieges bis zu 13000 Angestellte, davon allein in der R\&A zeitweise über 1000 Mitarbeiter. Unter dem »Teamchef« Langer sollten hier die grundsätzlichen Analysen für die politischen Entscheidungsträger der amerikanischen Kriegsbürokratie insgesamt und für die über 40 Abteilungen innerhalb der OSS-Administration angefertigt werden. In den nach regionalen Kriterien aufgebauten vier R\&A-Divisionen Europa-Afrika, UdSSR, Fernost und Lateinamerika waren unter anderem die erwähnten hochkarätigen amerikanischen und internationalen Akademiker beschäftigt, die neben der Analyse des nationalsozialistischen Herrschaftsregimes sowie Planungen über die Deutschlandpolitik nach Beendigung des Zweiten Weltkrieges sich auch und vor allem frühzeitig Gedanken machten und anspruchsvolle Arbeiten anfertigten über die von den Vereinigten Staaten betriebene komplexe, nicht allein auf den europäischen Kontinent bezogene Nachkriegspolitik. Im Zusammenhang mit der Politik der Vereinigten Staaten im Kontext des Kalten Krieges ist hier von zentraler Bedeutung, dass selbst in der Hochphase der militärischen Konfrontation mit dem nationalsozialistischen Gewaltsystem in der R\&A-Division UdSSR die politikstrategisch und wissenschaftliche Auseinandersetzung mit dem kommunistischen Staat unter Stalin weiterging. Dabei ist zu berücksichtigen, dass in den USA bereits seit den 1920er-Jahren die politische Auseinandersetzung mit der Sowjetunion auf intellektuelle und wissenschaftliche Beine gestellt worden war und hierbei mit Samuel Harper einer der renommiertesten Sowjetforscher des Landes gewonnen werden konnte. Des Weiteren existierte mit der sogenannten Russlandabteilung des State Department eine Gruppe von Sowjetexperten, die die Analyse mit dem russischen Kommunismus mit wissenschaftlichen Methoden betrieben. Wie für die R\&A insgesamt galt auch für die Regionalabteilung UdSSR, dass hier eine qualitativ bemerkenswerte Verquickung von Geheimdienstagentur und einer großen Beteiligung von intellektuellen Mitarbeitern bestand, für die eine politische, wissenschaftliche und primär analytisch ausgerichtete Forschung ihres Untersuchungsgegenstandes im Zentrum ihres OSS-Engagements stand.

\title{
1.6 Die "Kommunismusforschung « in der US-Administration nach dem Sieg gegen den Nationalsozialismus
}

Der Sieg der alliierten Truppen über den nationalsozialistischen Staat und die Kapitulation der deutschen Wehrmacht am 8. Mai 1945 bedeutete gleichfalls das Ende der R\&A als Abteilung des kriegsbedingten und -wichtigen OSS. Denn die Beendigung der kriegerischen Auseinandersetzungen führte zur Auflösung des donovanschen Geheimdienst- und Nachrichtenapparates im September 1945, was allerdings nicht gleichbedeutend war mit der restlosen Auflösung der Forschungsabteilung. Anlass war eine Verfügung des neuen US-Präsidenten Harry Spencer Truman, der noch während des Zweiten Weltkrieges die Führungsposition des Staates von dem am 12. April 1945 verstorbenen Roosevelt übernahm. Die Verfügung im September 1945 zählte zu einer der ersten Amtshandlungen von Truman. Während die Mitarbeiter der traditionellen, d.h. der genuin geheimdienstlichen Abteilungen zum War Department hinüberwechselten, erfolgte im Rahmen des Abbaus der riesengroßen Washingtoner Kriegsbürokratie die Übernahme der Forschungsabteilung R\&A durch das State De- 
partment. ${ }^{62}$ Eine weitere Konsequenz für die R\&A war, dass schätzungsweise 50 Prozent ihres Mitarbeiterstabes abgebaut wurden. ${ }^{63}$ In dem sich alsbald herauskristallisierenden und in den späteren 1940er-Jahren politisch, kulturell, ideologisch und auch militärisch zuspitzenden Ost-West-Antagonismus sollte die Forschungsabteilung, d. h. die Intellektuellen und Wissenschaftler, die sich mit der Sowjetunion sowie dem Kommunismus auseinandersetzten, einen zentralen Stellenwert besitzen.

Selbstverständlich lag der Hauptfokus nach dem Eintritt der Vereinigten Staaten in den Zweiten Weltkrieg auf dem bedingungslosen Kampf gegen das totalitäre nationalsozialistische Herrschaftsregime. Indessen hatte ebendiese mehrdimensionale Konfrontation mit Deutschland, d.h. der auf vielen Ebenen ablaufende totale Krieg, der eine in dieser Form historisch einmalige Konzentration der politischen, ökonomischen und militärischen US-amerikanischen Ressourcen zur Folge hatte, keineswegs das völlige Einstellen der Auseinandersetzung mit dem in dieser zugespitzten geschichtlichen Situation konsequenterweise als Koalitionspartner auftrumpfenden Sowjetunion. In der nach den vier operationswichtigen Weltregionen aufgeteilten R\&A des OSS erfolgte innerhalb der Division UdSSR die mit wissenschaftlichen Methoden durchgeführte Untersuchung des »Partners auf Zeit«. Zweifelsohne führte diese Forschungsabteilung insbesondere im Vergleich zur Central Europe Section ein Schattendasein, die sich infolgedessen mit wesentlich geringerer Mitarbeiterzahl zufriedengeben musste. Durch die sich nach Beendigung des totalen Krieges gegen Deutschland (bzw. die Achsenmächte) veränderten weltpolitischen Konstellationen avancierte die Abteilung Section on the Soviet Union - wie die Division UdSSR auch genannt wurde - alsbald zu einem zentralen Faktor innerhalb der US-Administration im Allgemeinen und dem State Department im Besonderen und erlangte sehr schnell einen beachtlichen Bedeutungszuwachs. Frühzeitig entwickelte sich hier ein ausgeprägtes Gefahrenbewusstsein hinsichtlich einer aktuellen sowjetischen Bedrohung für das westliche Staatenbündnis, sodass dort entscheidende Weichen auf die existenzielle und systemimmanente Konfrontation mit dem Kommunismus stalinistischer Provenienz und dem totalitären sowjetischen Staat unter dem Alleinherrscher Stalin gestellt wurden. Spätestens im Frühjahr 1947 war der Antikommunismus zu einem mächtigen Faktor der amerikanischen Realpolitik geworden.

In den hochstürmischen Zeiten des Zweiten Weltkrieges waren in der Forschungsabteilung des amerikanischen Geheimdienst OSS, wie gezeigt wurde, unter anderem nicht wenige Intellektuelle und Wissenschaftlicher aktiv, die sich wie beispielsweise Neumann oder Marcuse der internationalen politischen Linken verbunden fühlten. Gleichwohl hatten sich die Anhänger der linken Fraktion in der R\&A in den allermeisten Fällen einem expliziten »Anti-Antikommunismus« verpflichtet gefühlt, so die nachträgliche Einschätzung von H. Stuart Hughes, der zwischen 1943 und 1945 im OSS und, nachdem dieser aufgelöst wurde, von 1946 bis 1948 in der Forschungsabteilung des State Departement tätig war. ${ }^{64}$ Zum Beispiel hatte der Personenkreis um

62 Die sogenannten Deutschlandexperten, d. h. die Forschungsgruppe der emigrierten Intellektuellen um die anerkannte Führungsperson Neumann, wurden ebenfalls in das amerikanische Außenministerium übernommen - und zwar in die nachrichtendienstliche Abteilung.

63 Hierzu Näheres bei Söllner, Zur Archäologie der Demokratie in Deutschland, Bd. 2, S. 8 ff.

64 Zum Folgenden siehe insbesondere das Interview, in dem neben den biografischen Hinweisen sowie den individuellen Tätigkeiten im OSS und State Departement Hughes sehr aufschlussreich die 
Neumann, also die emigrierten jüdischen Linksintellektuellen, zu keinem Zeitpunkt irgendwelche Sympathien für die kommunistische Herrschaftsideologie unter Stalin, sodass neben der radikalen Gegnerschaft zum Nationalsozialismus auch eine tiefgehende Ablehnung gegenüber dem mörderischen stalinistischen Weltanschauungsregime zum politischen Konsens innerhalb der Intellektuellengruppe zählte. Zudem bestanden fundamentale Differenzen zum einen gegenüber den stalinistischen Intellektuellen nicht nur der Vereinigten Staaten und zum anderen gegenüber der KPUSA, die seit ihrer Gründung in den 1920er-Jahren unter der Kontrolle der Komintern respektive der KPdSU stand. Indes bestand aufseiten der antistalinistischen Linksintellektuellen im State Departement nach Kriegsende doch eine gewisse Blindheit gegenüber der Innen- und Außenpolitik der Sowjetunion, sodass in diesem Personenkreis eine nüchterne Kritik an der Politik Stalins ausblieb, was auch von Hughes im Nachhinein umstandslos eingeräumt wurde. Um sich nämlich nicht von dem nationalistischen antikommunistischen »Fieber « in den USA anstecken zu lassen, war die Grundposition ein expliziter »Anti-Antikommunismus«, so Hughes. Aus der zeithistorischen Perspektive der 1940er-Jahre ergab sich für den Personenkreis um Hughes im State Departement,

daß die ganze Wahrheit über den stalinistischen Terror noch nicht bekannt war, und vielleicht wollten wir sie nicht glauben. Wir waren natürlich überzeugt, daß es sich um ein tief autoritäres, terroristisches Regime handelte. [...] Wir glaubten, daß der Faschismus schlimmer sei als der Stalinismus. Denn, so Hughes weiter, »damals war schwer zu unterscheiden, was Propaganda war und was Tatsache. Ganz klar wurde es erst mit den Enthüllungen von Chruschtschow $1956 .{ }^{65}$

Die US-Regierung setzte bewusst die analytische Kraft der marxistischen und sozialdemokratischen Intellektuellen bzw. von der marxistischen Theorie inspirierten Wissenschaftler - in einigen Ausnahmen sogar Anhänger der stalinistischen Linken ${ }^{66}$ als politische Waffe gegen den Nationalsozialismus ein. Das Ende des Krieges und der schnell einsetzende Klimawechsel auch und vor allem im State Department hatten jedoch die sukzessive Entfernung von Personen aus der Washingtoner Bürokratie zur Folge, die als Stalinisten, Kommunisten oder Sozialisten bzw. als Sympathisanten der marxistischen und kommunistischen Idee verdächtigt wurden. ${ }^{67}$ Oberhand gewan-

institutionellen und »mentalen Veränderungen« des mehr und mehr um sich greifenden Klimas des Kalten Krieges im amerikanischen Außenministeriums nach Ende des Krieges schildert. Interview von Söllner mit Hughes: »Es war eine Sackgasse - und doch ist es wert, erinnert zu werden!« (Ebd., S. 46-58)

65 Ebd., S. 53.

66 Genannt werden müssen vor allem die beiden stalinistischen Ökonomen Paul A. Baran und Paul M. Sweezy, die nach ihrer Tätigkeit für die US-Regierung sehr bekannte und in Bezug auf die politische Linke einflussreiche theoretische Werke veröffentlichten. Im Zuge der aufkommenden KaltenKrieg-Atmosphäre wurden sie aus dem Regierungsapparat entfernt.

67 Natürlich gab es auch Ausnahmen. So zum Beispiel den frühen Mitarbeiter des Frankfurter Institutes für Sozialforschung und Anhänger der kommunistischen Bewegung Karl August Wittfogel. Das ehemalige KPD-Mitglied Wittfogel, der 1939 den Bruch mit der Partei vollzog, wurde vom State Departement in der Hochphase des Kalten Krieges als »Chinaexperte« engagiert und fertigte 1949 u. a. ein Memorandum über das kommunistische China unter Mao an. In New York arbeitete er am Institute 
nen grosso modo konservative Kräfte, die in Richtung eines militanten Antikommunismus gingen und denen eindeutig keine Sympathien für den sowjetischen Staat nachgesagt werden konnte.

\subsection{Der realistische Blick auf die sowjetische Außenpolitik unter Truman nach dem Ende der Anti-Hitler-Koalition}

Im Folgenden soll in aller Kürze auf den Verlauf des entstehenden Kalten Krieges eingegangen werden. Hierbei muss es ausreichen, auf einige entscheidende Etappenschritte der US-amerikanischen Politik sowie auf Stellungnahmen einflussreicher US-Diplomaten einzugehen, da die komplexe Geschichte der Ost-West-Blockkonfrontation vorausgesetzt werden kann. ${ }^{68}$ Dass mit dem Ende des siegreichen militärischen Kampfes gegen das nationalsozialistische Deutschland die systembedingte Auseinandersetzung mit dem existenziellen Gegner, d.h. dem sowjetischen Totalitarismus, weiterging, war nicht zuletzt den Kommunismusexperten im State Department um Kennan bewusst. Die Ost-West-Konfrontation war in den Jahren der Anti-Hitler-Koalition nur ruhiggestellt und spitzte sich nach 1945 immer mehr zu.

Die Kapitulation Deutschlands und das damit einhergehende Auseinanderbrechen der siegreichen Koalition zwischen den demokratischen Westmächten und der stalinistischen Sowjetunion hatte unter anderem zur Folge, dass mehr und mehr die völlig entgegengesetzten Gesellschaftsordnungen sowie politischen Ziele und ideologischen Vorstellungen der ehemaligen Partner ins öffentliche Bewusstsein drang. Obwohl

for Pacific Relations und war seit 1939 der Direktor des »Chinese History Project«an der Columbia University. Nachdem er sich schon in den 1920er-Jahren mit einigen Veröffentlichungen über China hervortat und nach der Verhaftung durch die Nazis in mehreren Konzentrationslagern »durch die Hölle des totalitären Terrors gegangen« war, emigrierte er 1934 vor dem Nationalsozialismus. Unter dem Pseudonym Klaus Hinrichs veröffentlichte Wittfogel in London den Roman Staatliches Konzentrationslager VII. Weithin bekannt wurde er insbesondere durch den Klassiker der Totalitarismusforschung Oriental Despotism (New Haven [NY] 1957; dt. Ausg.: Die Orientalische Despotie. Eine vergleichende Untersuchung totaler Macht, Köln 1962). Ein weiterer prominenter Linksintellektueller, der vom amerikanischen Staat weiter beschäftigt wurde, war Herbert Marcuse, der nach der Auflösung des OSS von 1945 bis 1953 vom State Departement als sogenannter Principal research analyst for Germany übernommen wurde. In diesem Zusammenhang legte er als Leiter einer Forschungsgruppe einer Spionageabwehrabteilung des amerikanischen Außenministeriums in Washington eine materialgesättigte Analyse der internationalen Entwicklungspotenziale des kommunistischen Systems vor; hierbei kam er zu dem Ergebnis, dass die kommunistischen Parteien in Westeuropa entweder integriert oder bedeutungslos seien und angesichts einer möglichen wirtschaftlichen Krise davon ausgegangen werden könnte, dass nicht ein kommunistischer Umsturz zu erwarten sei, sondern ein neuer Faschismus. Im Rahmen seiner Tätigkeiten für das State Departement nutzte Marcuse vorhandene Freiräume, um mit Vorarbeiten für sein späteres Buch über den Sowjetmarxismus zu beginnen. Das Forschungsergebnis erschien unter dem Titel Soviet Marxism. A Critical Analysis 1958 in New York (dt. Ausg.: Die Gesellschaftslehre des Sowjetischen Marxismus, Neuwied/Berlin 1964). Interessanterweise bedankte sich Marcuse in dem Buch ausdrücklich bei dem Direktor des Russian Research Center, William L. Langer, der ihm die Möglichkeit für Recherchen in der Institution für den Forschungsband bot. Langer war Marcuses höchster Vorgesetzter als Leiter der OSS-Behörde während der Mitarbeit in der R\&A.

68 Vgl. exemplarisch die beiden Standardwerke von Wilfried Loth, Die Teilung der Welt: Geschichte des Kalten Krieges 1941-1955, München 1987 und Bernd Stöver, Der Kalte Krieg 1947-1991. Geschichte eines radikalen Zeitalters, München 2007. 